\title{
Saving Digital Humanities
}

\author{
„LANGZEITARCHIVIERUNG BY DESIGN “
}

Richard Hörmann \& Daniel Schlager

Universität Salzburg, IT Services

richard.hoermann@sbg.ac.at, daniel.schlager@sbg.ac.at

Keywords: Long-Term Archiving, IT-Infrastructure, Repository, Stefan Zweig digital, EbnerOnline, MHDBDB

\begin{abstract}
Archiving Digital Humanities projects without expiration dates is one of the biggest challenges which this young discipline faces. To meet this challenge, the University of Salzburg is building a platform on which digital containers ensure the safe storage of projects.
\end{abstract}

\section{Konzeption von dhPLUS (Richard Hörmann)}

Der Aufbau einer Plattform dhPLUS für die Langzeitarchivierung [LZA] von Digital Humanities [DH] Projekten an der Paris-Lodron-Universität-Salzburg [PLUS] geschieht im Rahmen eines 3-jährigen Projektes, das aus HRSM Strukturmitteln des österreichischen Wissenschaftsministeriums finanziert wird. Das Kompetenznetzwerk Digitale Edition [KONDE] genannte Projekt wird von der Universität Graz geleitet, die PLUS ist einer von mehreren österreichischen Partnern. Vom Rektorat der PLUS wurden die IT-Services der Universität Salzburg [ITS] beauftragt, das Projekt KONDE PLUS durchzuführen. Um die Plattform von Beginn an den Anforderungen der Zielgruppe entsprechend entwickeln zu können, wurden drei Pilotprojekte (Ferdinand Ebner: Gesammelte Werke. Online Edition [EbnerOnline] http://wfe.sbg.ac.at/, Mittelhochdeutsche Begriffsdatenbank [MHDBDB] http://mhdbdb. sbg.ac.at/, Stefan Zweig digital [SZD] http://www.stefanzweig.digital/) definiert, deren Migration auf die Plattform während der Projektlaufzeit vorgenommen wird. Dementsprechend setzt sich das Team aus Vertretern dieser Pilotprojekte und aus Mitarbeiter*innen der IT-Services zusammen, die auch den Leiter des gesamten Projektes stellen.

Das in der Planungsphase von dhPLUS entwickelte Konzept beinhaltet eine Reihe von SchlüsselParametern, die wie folgt formuliert wurden:

Schlüssel-Parameter der Plattform:

- Organisation: Die Plattform dhPLUS muss als ein Service der ITS der PLUS eingerichtet werden. Spätestens bei Start des Regelbetriebes müssen die ihr zugeordneten Stellen entfristet und aus dem Budget der ITS bzw. der PLUS finanziert werden (d.h. keine Drittmittel-Stellen). Die Plattform muss in die IT-Infrastruktur der ITS eingebunden werden, insbesondere an das vorhandene Datenbank- und Archivsystem. 
- Dokumentation: Die Plattform und ihre Projekte sind umfangreich zu dokumentieren. So wird gewährleistet, dass die trotz auftretenden und nicht prognostizierbaren Fluktuationen bezüglich Personal, Software etc. langfristig effizient bewältigt werden können.

- Daten-Sicherheit: Die Sicherheit der Daten ist in dreifacher Hinsicht zu garantieren: einmal in physikalisch-technischer mit „bitstream preservation“ Maßnahmen der lokalen IT-Infrastruktur. Zum zweiten bezüglich der Daten-Formate: Es sind standardisierte Formate zu verwenden, die die zukünftige Weiterverwendung, den Austausch und die Umstellung auf neue Formate ermöglichen. Und zum dritten bezüglich der Betriebssicherheit: Auch bei Ausfall von Teilen des Systems oder beim Austausch von Komponenten soll der Zugriff auf die Daten gewährleistet bleiben.

- Code-Sicherheit: Die in den DH-Projekten verwendete Software ist genauso zu sichern wie die Daten.

- Identifikation: Den digitalen Ressourcen der Plattform sind persistente Identifikatoren (PID) zuzuordnen, die eine eindeutige Identifikation der Ressourcen über ihre gesamte "Lebenszeit“ gewähren.

- Beschreibung: Die Daten der DH-Projekte auf der Plattform sind mit Metadaten zu ergänzen, die die Daten beschreiben und ihre Sicherung, Klassifikation, Weiterverarbeitung (Suche) und Wiederverwendbarkeit ermöglichen.

- Versionierung: Der Zugriff auf historische Versionen der Projekt-Ressourcen muss Teil der LZA-Strategie der Plattform sein.

- Bearbeitung: Die Plattform hat eine Archiv- und Bearbeitungsfunktion, d.h. sie hat nicht nur der Sicherung der Projektressourcen zu dienen, sondern genauso deren ständiger Bearbeitung, damit Ressourcen erweitert, korrigiert und optimiert werden können.

Bei der Erstellung von Modellen und Strategien zur Einhaltung und Umsetzung dieser Schlüsselparameter gab es zwei Leitideen: einerseits möglichst wenig dort zu erfinden, wo bereits Modelle oder Standards vorhanden sind und zum anderen dort innovativ zu sein, wo die bestehenden Lösungen unzureichend sind.

Ein Beispiel ist die Basis-Struktur der Plattform: obwohl von den Schlüssel-Parametern nicht vorgegeben, wurde auf das international anerkannte Open Archival Information System [OAIS]-Referenzmodell für offene Archivsysteme zurückgegriffen: 


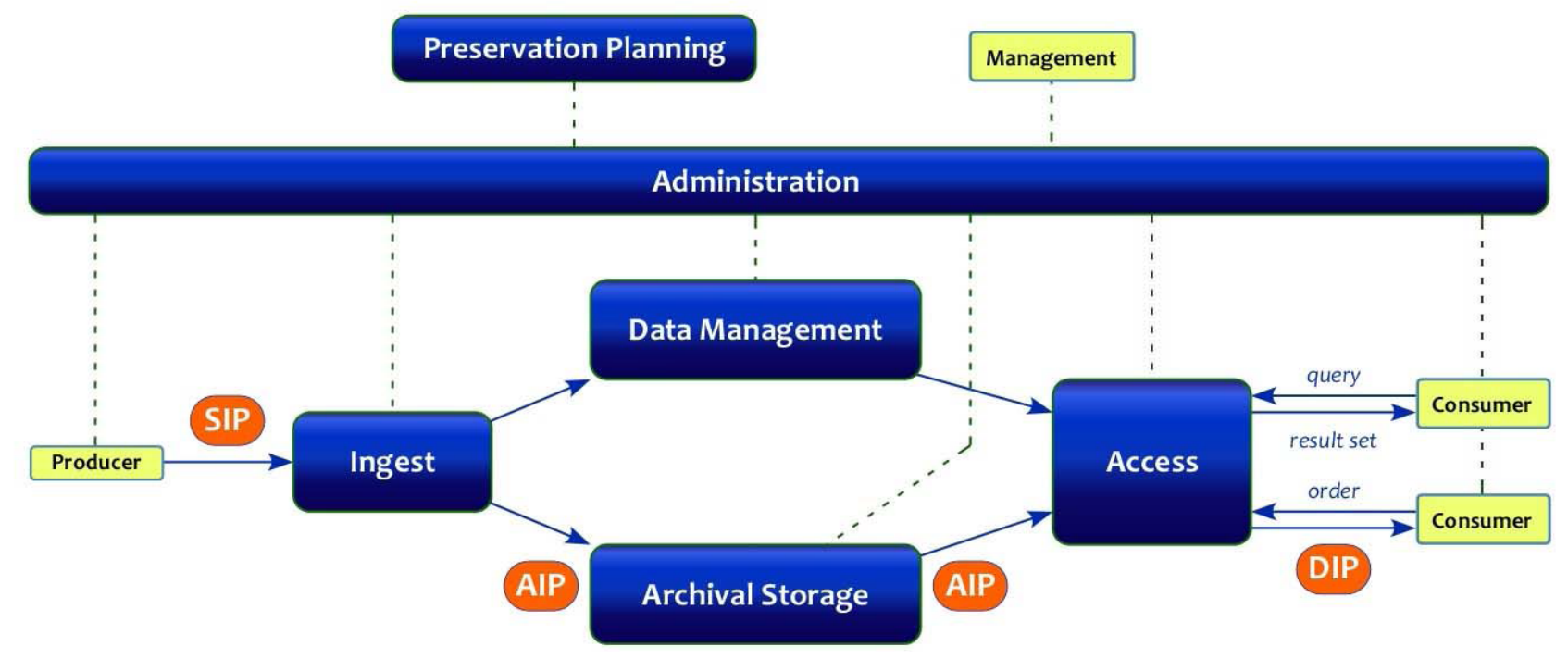

Abb. 1: OAIS Referenzmodell

Dem OAIS nach hängt eine erfolgreiche Archivierung digitaler Ressourcen davon ab, ob im System Informationspakete strukturiert vorliegen (Archival Information Package [AIP], Submission Information Package [SIP], Dissemination Information Package [DIP]), bestimmte Funktionen implementiert sind (Ingest, Archival Storage, Data Management, Access, Administration, Preservation Planning) und Rollen klar definiert sind (Producer, Management, Consumer).

Im OAIS Modell sind es keine Daten oder Ressourcen, die eingegeben, archiviert oder ausgegeben werden, sondern Informationen. Es gibt zwei Arten von Informationen, die in Informationspaketen [IP] abgelegt werden:

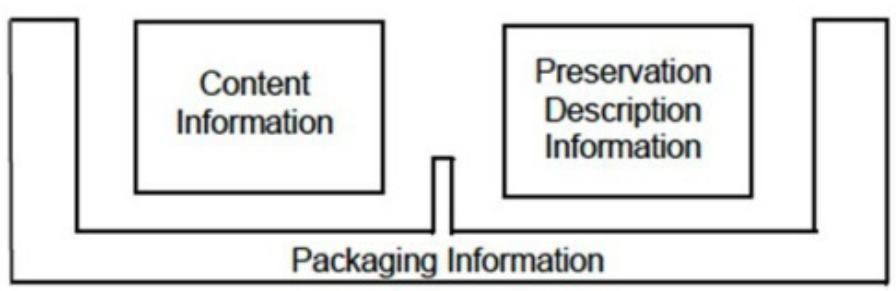

Abb. 2: OAIS Modell

1. Inhaltliche Informationen, in den digitalen Ressourcen repräsentiert als Projektdaten, die erhalten werden sollen: Content Information.

2. Informationen, die zur Erhaltung der inhaltlichen Informationen erforderlich sind: Preservation Description Information [PDI].

SIP, AIP und DIP sind Typen solcher IP, die sich bezüglich ihrer Position im Workflow des Systems unterscheiden:

- SIP sind Pakete, die vom Producer im Ingest an das System übergeben werden.

- AIP sind Pakete, die im System gespeichert und verwaltet werden.

- DIP sind Pakete, die vom Consumer aus dem System entnommen werden. 
OAIS ist ein Modell, in dessen Zentrum die Archivierung von Informationen steht. Da die Plattform dhPLUS aber für spezielle Projekte, nämlich Projekte der $\mathrm{DH}$, gebaut wird, ist es sinnvoll zu untersuchen, ob sich aus der Perspektive dieser Art von Projekten zusätzliche Anforderungen ergeben.

Obwohl DH-Projekte unter sich sehr heterogen sind und aufgrund der unterschiedlichen Projektziele, -methoden, technischen Realisierungen etc. auf allen Ebenen Singularitäten aufweisen, lassen sich doch aus den meisten Projekten grundlegende Gemeinsamkeiten abstrahieren.

Ein Modell, das diese Gemeinsamkeiten gut abbildet, ist das von der Berlin-Brandenburgischen Akademie der Wissenschaften [BBAW] vorgestellte Layer-Modell für Digitale Editionen:

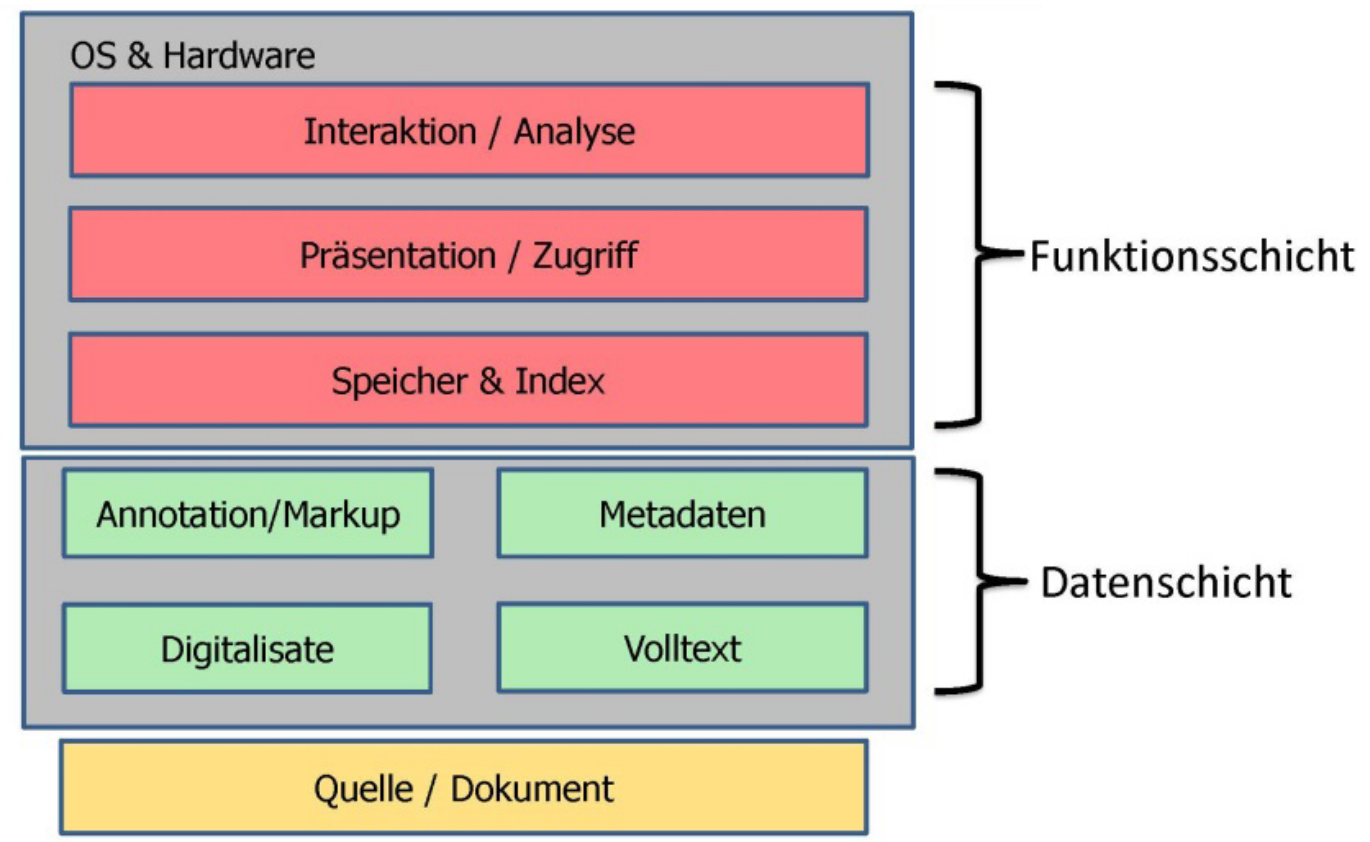

Abb. 3: Layer-Modell für Digitale Editionen

Diesem Modell zufolge besteht ein DH-Projekt aus zwei basalen Schichten: der Daten- und der Funktionsschicht. Sollen die Ergebnisse von DH-Projekten ohne Ablaufdatum gesichert werden, sind demnach nicht nur ihre Daten, sondern auch ihre Funktionen zu sichern.

Ein Schlüssel-Parameter für die LZA ist das Vorliegen einer auf dem aktuellen Stand gehaltenen, umfangreichen Dokumentation sowohl der Plattform als auch der einzelnen Projekte auf ihr. Da dieser Aspekt der LZA im Layer Modell der BBAW nicht berücksichtigt ist, ist das Layer Modell von dhPLUS um eine Dokumentations-Schicht zu ergänzen.

Aus dem erweiterten Layer-Modell folgt auch ein erweitertes OAIS-Modell für dhPLUS: zu den inhaltlichen Informationen der IP zählen jetzt neben den Informationen der Datenschicht auch die Informationen aus der Funktions- und Dokumentationsschicht.

OAIS- und Layer-Modell von dhPLUS sind abstrakte Modelle, die ein vermittelndes Konzept erfordern, das ihre SW-seitige Realisierung ermöglicht. Aus dem pragmatischen Ansatz, möglichst viel von bestehenden Lösungen zu übernehmen, wurde für dhPLUS ein ObjektModell ausgewählt, das im Geisteswissenschaftlichen Asset Management System [GAMS] der Universität Graz verwendet wird und seinerseits auf einem Fedora-Modell basiert. Das daraus für dhPLUS modellierte Digitale Objekt [DO] ist so aufgebaut: 


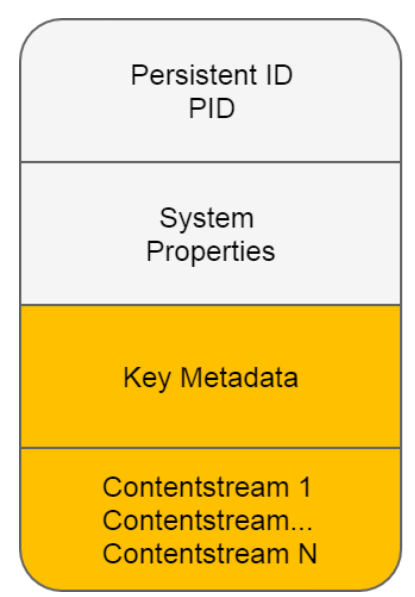

Abb. 4: Digitales dhPLUS-Objekt

Ein DO besteht aus vier Teilen: einem Teil mit dem eindeutigen Identifier des Objektes, einem zweiten mit den Systemeigenschaften des Digitalen Objektes, einem dritten mit den Schlüssel-Metadaten, und einem vierten mit den zu sichernden Inhalten des Objektes.

Systemeigenschaften und Schlüssel-Metadaten des DO unterscheiden sich insofern als erstere Metadaten sind, die Informationen über das DO in Bezug auf das Plattform-System beinhalten, während letztere Informationen über die Inhalte des DO (= Contentstreams) bereitstellen.

Dieses Modell eines DO erfüllt sowohl die Schlüsselparameter als auch die Anforderungen, die sich aus dem erweiterten OAIS und Layer-Modell von dhPLUS ergeben.

Die drei Schichten des Layer-Modells führen zu drei verschiedenen Typen von DO, die sich in Bezug auf die Contentstreams unterscheiden:

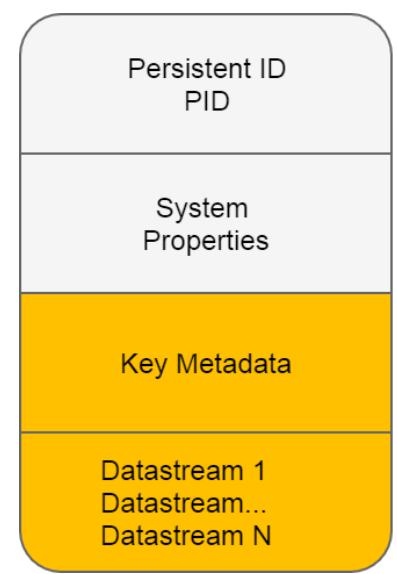

Abb. 5: Digitales Daten Objekt

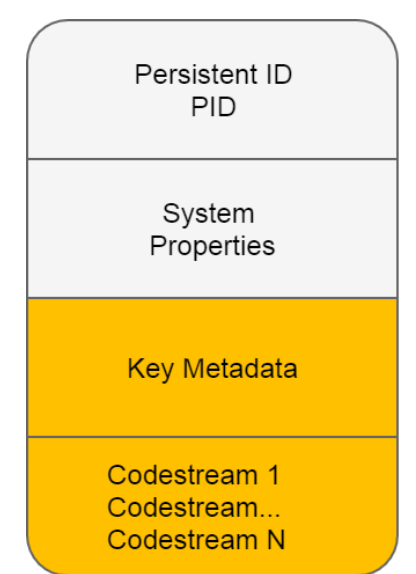

Abb. 6: Digitales Funktions Objekt

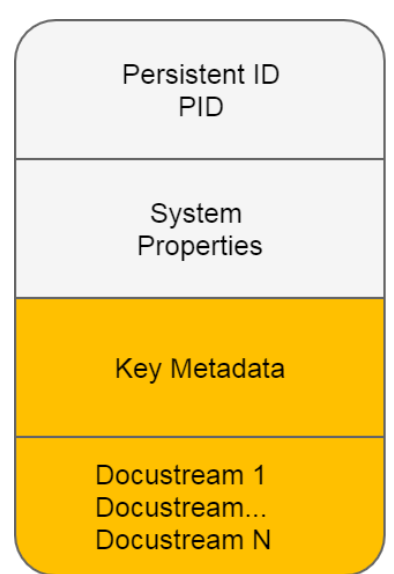

Abb. 7: Digitales Dokumentations Objekt

Ein Digitales Daten Objekt [DDO] enthält als Contentstreams Datastreams mit den Daten und Metadaten der Datenschicht, ein Digitales Funktions Objekt [DFO] enthält Codestreams mit den Funktionen und Services der Funktionsschicht und ein Digitales Dokumentations Objekt [DDCO] Docustreams, deren Contentstreams der Dokumentationsschicht entnommen sind.

Die in jedem IP des OAIS-Modells enthaltene PDI wird in den Key-Metadaten des DO modelliert. Die systemrelevanten Informationen für die Archivierung, das Datenmanagement und die Administration werden in den System Properties abgelegt. 
Mit der Persistent ID jedes Objektes ist der Schlüssel-Parameter der eindeutigen Identifikation jeder Projekt-Ressource gegeben.

\section{Technische Realisierung von dhPLUS (Daniel Schlager)}

Die persistente ID entspricht dem Universally Unique Identifier [UUID] Standard der Version 5. Bei dieser namensbasierten UUID wird aus einem DNS Namen eine UUID gebildet. Der Vorteil gegenüber der (Pseudo-)zufällig generierten UUID (Version 4) ist die garantierte Eindeutigkeit durch den einberechneten DNS Namen des Repositoriums und der Projekt-ID.

Die Projekt-ID entspricht einem Namenskürzel für ein spezifisches Projekt. Als Beispiel kann hier das Kürzel: MHDBDB angeführt werden.

Daraus errechnet sich folgende UUID: e6921bb7-212f-5922-9a62-f6e24f06669a

Aus dieser UUID wird eine URI erzeugt, die eine Ressource eindeutig identifiziert:

https://dhplus.sbg.ac.at/rest/MHDBDB/e6921bb7-212f-5922-9a62-f6e24f06669a

Ressourcen im dhPLUS Repository werden in RDF modelliert. Als Basisformat wird RDF/XML verwendet. Die XML Dokumente werden zur automatischen Verarbeitung in einer Oracle Datenbank gespeichert (und auch dort archiviert). Der Einsatz von Oracle als Datenbank und Archivierungssystem wurde aus strategischen Gründen gewählt. Grundsätzlich ist die dhPLUS Software modular und unabhängig von spezifischen Datenbanksystemen aufgebaut. Die Oracle Plattform, bestehend aus der Oracle Datenbank und dem Oracle Archivsystem, sind das Kernstück der IT Services der Universität Salzburg. Dies ermöglicht dhPLUS die einfache, kosteneffektive und sichere Implementierung und Umsetzung einer LZA-Strategie.

Durch den konsequenten Einsatz von RDF - nicht nur zur Modellierung der digitalen Objekte, sondern auch für die Projektkonfigurationen und andere Daten - sprechen wir bei dhPLUS von einem RDF bzw. Knowledge Graph basierten Repository.

Die RDF/XML Dokumente werden vom System in einem Triple Store, wofür aktuell Apache Jena eingesetzt wird, weiterverarbeitet und mittels einer REST-API und einem SPARQL-Endpoint zur Verfügung gestellt. Durch die Modularität und Aufteilung der API in Microservices ist ein Austausch des Triple Stores jederzeit möglich. 


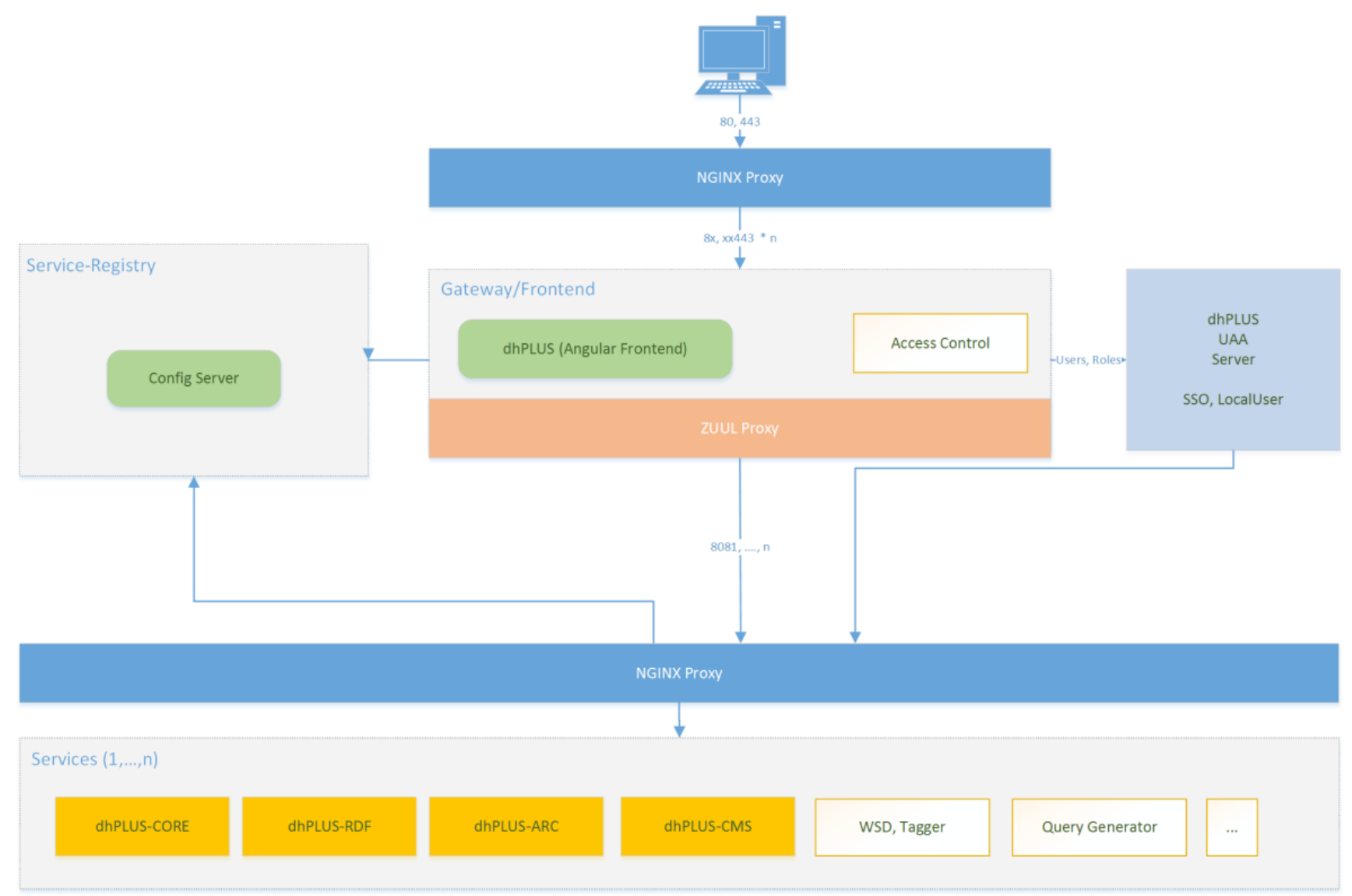

Abb. 8: Microservice Architektur dhPLUS

Ein weiterer Vorteil von Microservices gegenüber einem monolithischen Ansatz ist die leichtere Skalierbarkeit und dahingehend auch die hohe Verfügbarkeit.

In Abb. 8 ist zu sehen, dass jeglicher Zugriff auf einen Service und auf das vom Frontend zur Verfügung gestellte Gateway über einen Proxy gehandelt und verteilt wird. Zusätzlich zu diesem NGINX Proxy beinhaltet der Gateway Dienst einen eigenen Proxy (ZUUL), welcher die ankommenden API Requests an den zuständigen, in der Service-Registry registrierten Service weiterleitet.

Die API, welche die grundlegenden RDF Funktionalitäten für die Plattform bereitstellt, ist in einem eigenen Microservice (dhPLUS-RDF) verankert und nahezu 100\% kompatibel zu Fedora 4. Mit dieser Emulationsschicht kann ein Austausch mit anderen auf Fedora basierten Repositorien gewährleistet werden. 


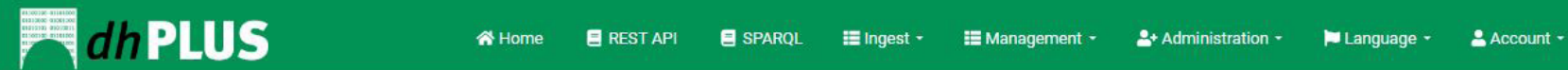

Digital Object

\begin{tabular}{|c|c|}
\hline OBJECT PROPERTIES & DOWNLOAD BINARY \\
\hline \multicolumn{2}{|l|}{ lastModified } \\
\hline \multicolumn{2}{|l|}{ Children 0} \\
\hline PROPERTIES & - \\
\hline \multicolumn{2}{|l|}{$\begin{array}{l}\text { lastModified } \\
\text { 2019-04-30T08:49:12.264Z }\end{array}$} \\
\hline $\begin{array}{l}\text { ProjectName } \\
\text { MHDBDB }\end{array}$ & UPDATE PROPERTIES \\
\hline $\begin{array}{l}\text { filename } \\
\text { SQL-injections.zip }\end{array}$ & Update Properties \\
\hline $\begin{array}{l}\text { hasMimeType } \\
\text { application/zip }\end{array}$ & $\begin{array}{l}\text { > <http://www.w3.org/1999/02/22-rdf-syntax-ns\#ype> } \\
\text { <http://www.w3.org/ns/ldp\#\#Resource>. }\end{array}$ \\
\hline \multicolumn{2}{|l|}{ rdf: type } \\
\hline \multicolumn{2}{|l|}{ http://www.w3.org/ns/ldp\#Resource } \\
\hline \multicolumn{2}{|l|}{ http://www.w3.org/ns/ldp\#NonRDFSource } \\
\hline \multicolumn{2}{|l|}{ http://www.w3.org/ns/ldp\#Binary } \\
\hline http://www.w3.org/ns/dp\#\#NonRdfSourceDescription & UPDATE \\
\hline http://www.loc.gov/premis/rdf/v1\#Object & \\
\hline
\end{tabular}

Abb. 9: REST-API dhPLUS

Abb. 9 zeigt das Beispiel eines Digitalen Daten Objektes [DDO] so wie es sich beim gegenwärtigen Stand von KONDE PLUS als Antwort auf einen REST-API Request im Browser darstellt. Zu sehen ist die PID des Beispiel-DDO (https://dhplus-dev.sbg.ac.at/rest/MHDBDB/8fb0d8ac-eb20-4dd7bdaf-66298e26e534), seine Object (= System) Properties und die Properties (= Key Metadata) der

Datastreams. Wie am Dateinamen ersichtlich handelt es sich bei den Datastreams um mehrere gezippte Dateien, die über den Button „Download Binary“ geöffnet werden können.

\section{Literaturverzeichnis}

Consultative Committee for Space Data Systems [CCSDS], Hg., Reference Model for an Open Archival Information System (OAIS), Washington D. C. 2012, 2-6, online unter: https://public.ccsds.org/pubs/650x0m2.pdf (13.05.2019).

Alexander Czmiel, Dokumentation, Werkzeugkasten, Pakete. Nachhaltigkeit von Daten und Funktionalität Digitaler Editionen, in: Konferenzabstracts. DHd2017 Bern. Digitale Nachhaltigkeit, Bern 2017, 138-141, online unter: http://www.dhd2017.ch/wp-content/uploads/2017/02/Abstractband_ergaenzt.pdf (13.05.2017).

OAIS-Based Processes, online unter: https://www.icpsr.umich.edu/icpsrweb/content/datamanagement/lifecycle/ oais.html (07.06.2019.)

Elisabeth Steiner / Johannes Stigler , GAMS and Cirilo Client. Policies, documentation and tutorial, Graz 2014 (Letzte Aktualisierung 10.04.2017), online unter: hdl.handle.net/11471/521.1.1 (13.05.2019). 